\title{
Produtividade dos laboratórios de prótese dentária do SUS no estado da Bahia
}

\author{
Productivity of SUS dental prosthesis \\ laboratories in the state of Bahia
}

\section{Francisvânia Camilo Santos Alves ${ }^{1}$ \\ Lília Paula de Souza Santos ${ }^{2}$ (C) Natally Rocha Oliveira 3 (1) Luíza Eloy Guimarães ${ }^{4}$}

\begin{abstract}
1,4Faculdade Maria Milza (Governador Mangabeira). Bahia, Brasil. francys.odontofamam@gmail.com, luiza_eloy@hotmail.com 2Instituto de Saúde Coletiva da Universidade Federal da Bahia (Salvador). Bahia, Brasil. lilia_paula@yahoo.com.br ${ }^{3}$ Autora para correspondência. Faculdade Maria Milza (Governador Mangabeira). Bahia, Brasil. natallyrocha.oli@gmail.com
\end{abstract}

RESUMO | INTRODUÇÃO: A dificuldade no acesso aos serviços de saúde bucal e a prática odontológica mutiladora acarretou em um número elevado de indivíduos acometidos pelo edentulismo. Com o intuito de reverter este quadro, foi implantada a Política Nacional de Saúde Bucal e com ela o Laboratório Regional de Prótese Dentária. OBJETIVO: Descrever a produtividade de prótese dentária destes laboratórios no estado da Bahia no ano de 2016. METODOLOGIA: Foi realizado um estudo quantitativo-descritivo, foram coletados dados provenientes do Sistema de Informação Ambulatorial do Sistema Único de Saúde relativos a produtividade de prótese dentária. As informações referentes à estrutura física, recursos humanos dos laboratórios e a distribuição destes por regiões de saúde foram coletadas no Cadastro Nacional de Estabelecimentos de Saúde e as demais informações sobre o município no Instituto Brasileiro de Geografia e Estatística. Foram realizadas a frequência absoluta e relativa das variáveis, e a comparação da produtividade com as características estruturais dos laboratórios e das regiões de saúde da Bahia. RESULTADOS: Foram analisados 51 municípios que oferecem os serviços de prótese dentária no SUS, destes 32 possuem CEO. Observou-se um total de 46.870 próteses produzidas do tipo prótese total, parcial removível, fixa e sobre implante, sendo que as cidades com CEO produziram $66,49 \%$ e os 19 municípios que não têm CEO produziram $33,51 \%$. O tipo de prótese mais realizado foi a prótese total $(58,73 \%)$, seguido pela prótese parcial removível $(40,07 \%)$. A região Sul apresentou a maior produtividade de próteses dentárias $(24,26 \%)$ enquanto a região Centro Norte produziu a menor quantidade (2,28\%). CONCLUSÃO: Embora haja uma quantidade considerável de próteses produzidas, apenas $12,2 \%$ dos municípios baianos possuem laboratório de prótese dentária, sendo necessários expansão e fortalecimento deste programa.

PALAVRAS-CHAVE: Serviços de saúde bucal. Prótese dentária. Avaliação de serviços de saúde.
ABSTRACT | INTRODUCTION: The difficulty in accessing oral health services and the practice of dental mutilation has led to a high number of individuals affected by edentulism. In order to reverse this situation, the National Oral Health Policy was implemented and with it the Dental Prosthesis Laboratories. OBJECTIVE: To evaluate the productivity of the dental prothesis of these laboratories in the state of Bahia, in 2016. METHODOLOGY: This was a quantitative-descriptive study in which data were collected from Outpatient Information System of the Unified Health System regarding dental prosthesis productivity. The information of physical structure, human resources of the laboratories and their distribution by health regions were collected in the site of the National Registry of Health and other information about the municipality in the Brazilian Institute of Geography and Statistics. The absolute and relative frequency of the variables and the comparison of productivity with the structural characteristics of the laboratories and health regions of Bahia were presented. RESULTS: We analyzed 51 municipalities that offer dental prosthesis services in SUS, of these 32 have CEO. A total of 46,870 prostheses produced of the type dentures, partial removable, fixed and on implant were observed, with the cities with CEO producing $66.49 \%$ and the 19 cities without CEO produced $33.51 \%$. The type of prosthesis most performed was the total (58.73\%) followed by the removable partial prosthesis $(40.07 \%)$. The South region presented the highest productivity of dental prostheses (24.26\%) while the region Center North produced the lowest amount (2.28\%). CONCLUSION: Although there is a considerable amount of prostheses produced, only $12.2 \%$ of the municipalities in Bahia have a dental prosthesis laboratory, even demonstrating a considerable amount of productivity.

KEYWORDS: Dental health services. Dental prosthesis. Health Services research. 


\section{Introdução}

No Brasil, o último levantamento epidemiológico nacional de saúde bucal mensurou o edentulismo pelo uso e necessidade de prótese dentária, sendo que $13,7 \%, 68,8 \%$ e $92,7 \%$ da população brasileira, respectivamente, na faixa etária de 15 a 19, 35 a 44 e 65 a 74 anos, apresentavam necessidade de prótese dentária]. Verificou-se com base no resultado que diversos fatores podem influenciar esta condição, dentre eles: variáveis socioeconômicas, demográficas e o uso de serviços de saúde bucalf.

A perda dentária é um dos principais problemas de saúde bucal na população, por conta da elevada prevalência e dos danos funcionais consequentes, tornando-se um problema de saúde pública. Os dentes possuem funções fundamentais em vários aspectos: aparência facial, fonética, mastigação e deglutição, e a sua ausência influencia negativamente na qualidade de vida das pessoas. Por este motivo, é necessária a reabilitação oral para a recuperação parcial ou total das capacidades funcionais perdidas l $^{2}$

O Ministério da Saúde em 2004 implantou a Política Nacional de Saúde Bucal. Esta se constitui em uma série de medidas que garantem ações de promoção, prevenção e recuperação da saúde bucal dos brasileiros, fundamental para a saúde geral e qualidade de vida da populaçãof. Entre as ações deste programa estão: a inclusão dos procedimentos voltados a fase clínica da instalação de próteses dentárias na atenção básica e a implantação dos Laboratórios Regionais de Próteses Dentárias (LRPD) para a confecção de próteses dentárias pelo Sistema Único de Saúde (SUS)

Os LRPD são estabelecimentos cadastrados no Sistema de Cadastro Nacional de Estabelecimentos de Saúde como Unidade de Serviço de Apoio Diagnóstico Terapêutico para realizar, no mínimo, os serviços de prótese dentária total e prótese parcial removível. No início da criação desta política, para o credenciamento destes estabelecimentos era utilizado um parâmetro populacional, em que, para cada grupo de 500.000 habitantes poderia ser cadastrado um LRPD por região de saúde. Em 2009, este parâmetro deixou de existir e os municípios com qualquer base populacional puderam requerer o credenciamento, sem restrições quanto à natureza jurídica dos estabelecimentos:
No ano de 2018 havia o cadastro de 1.758 LRPD, apresentando uma produtividade de 736.970 próteses no país no mesmo períodol. A oferta de próteses pelo serviço público odontológico vem aumentando e com ela também é possível notar avanços nas ações da PNSB como um todo, entretanto, é possível notar fragilidades na sua institucionalização|⿴囗十)

Pesquisas demonstram desigualdades na distribuição geográfica dos serviços de prótese dentária pelo SUS 1 国 e seu acesso ainda é restrito levando em consideração a demanda populacional por reabilitação protétican 14. Dados do Programa de Melhoria do Acesso e da Qualidade da Atenção Básica apresentam que apenas 43\% das Equipes de Saúde Bucal (ESB) no país realizam procedimentos de prótese dentária, e a maior proporção delas está localizada nas regiões Nordeste $(33,9 \%)$ e no Sudeste $(33,6 \%)$. Entretanto, ainda não existem publicações específicas deste serviço para o estado da Bahia.

Nesta perspectiva, este trabalho teve como objetivo descrever a produtividade de próteses dentárias dos Laboratórios Regionais de Prótese Dentária do Sistema Único de Saúde no estado da Bahia no ano de 2016.

\section{Metodologia}

Foi realizado um estudo quantitativo-descritivo utilizando dados secundários provenientes do Departamento de Informática do SUS (DATASUS) e do Instituto Brasileiro de Geografia e Estatística (IBGE).

Os LRPD da Bahia participaram do estudo. Como critério de inclusão para o estudo foram admitidos os laboratórios implantados até o ano de 2015 e com produção ambulatorial no ano de 2016. Foram excluídos os laboratórios não cadastrados até o período descrito. Os dados secundários utilizados foram relativos à quantidade aprovada de procedimentos odontológicos ambulatoriais do LRPD e sua estrutura física, referentes ao ano de 2016.

Os dados sobre os procedimentos ambulatoriais foram coletados no Sistema de Informação Ambulatorial do SUS, utilizando-se os seguintes critérios: informações de saúde (TABNET), assistência à 
saúde e produção ambulatorial por local de atendimento. Foram empregados também os seguintes filtros: procedimento, quantidade aprovada, período e subgrupo de procedimentos.

Os códigos de produção ambulatorial escolhidos foram aqueles relacionados ao monitoramento da produção ambulatorial proposta pela Nota Técnica 24 da Coordenação Geral de Saúde Bucal do Ministério da Saúde ${ }^{15}$, a partir dos códigos 0701070129 para prótese total mandibular e 0701070137 para prótese total maxilar; código 0701070145 para próteses coronárias/intrarradiculares fixas/adesivas (por elemento); códigos 0701070099 para prótese parcial mandibular removível; 0701070102 para prótese parcial maxilar removível e 0701070153 para prótese sobre implante.

No site do Cadastro Nacional de Estabelecimentos de Saúde (CNES) foi realizada a coleta de informações relativas a presença ou não de Centro de Especialidade Odontológica (CEO) no município com LRPD e informações sobre os recursos humanos dos LRPD (presença de cirurgião-dentista (CD) e/ou técnico de prótese dentária (TPD), seguindo filtros: acesso rápido, consulta estabelecimento - identificação, atende SUS, estado (Bahia), pesquisar laboratório de prótese, ficha de estabelecimento, profissionais.

Os dados referentes a população e ao Índice de Desenvolvimento Humano (IDH) dos municípios com LRPD foram coletados no site do IBGE, utilizando os seguintes filtros: estatísticas, por cidade e estado, seleciona o estado Bahia e o município relacionado.

Os dados da produção ambulatorial de prótese dentária foram tabulados por meio do software TabWin.
Após a tabulação, eles foram exportados para o programa Microsoft Office Excel (versão 2016) onde foi realizada a categorização dos procedimentos e a distribuição das frequências absolutas e relativas de cada variável utilizada no estudo e a comparação entre a produtividade dos municípios com e sem CEO e por região do estado da Bahia.

\section{Resultados}

De um total de 417 municípios no estado da Bahia, foram encontrados 51 (12,2\%) municípios que oferecem serviços de prótese dentária no SUS no ano de 2016. Neste período foi registrado um total de 46.870 próteses produzidas de acordo com os códigos de produção ambulatorial. Do total de 51 laboratórios, $15(29,4 \%)$ possuíam Técnico de Prótese Dentária Cadastrado, e apenas 3 (5,9\%) possuíam um ou dois cirurgiões-dentistas cadastrados. O IDH não diferenciou muito entre os municípios analisados, apresentando valor médio de 0,632 (+/-0,06). A média populacional dos municípios foi de 135.220 (+/-415.216) com um valor máximo de 2.953 .986 (Salvador) e mínimo de 4.456 (Maetinga). Os valores populacionais e de IDH encontrados não interferiram nos valores de produtividade das próteses.

A tabela 1 apresenta a distribuição dos laboratórios por regiões da Bahia. Nota-se que todas as regiões da Bahia possuem LRPD, com maior quantidade na região Leste $(13,7 \%)$ e menor nas regiões Nordeste $(2,0 \%)$ e Norte $(2,0 \%)$. As regiões Sul e Leste apresentaram maior produtividade de próteses dentárias, respectivamente $24,3 \%$ e $23,5 \%$.

Tabela 1. Quantidade de laboratórios regionais de prótese dentária e produção de prótese dentária por regiões da Bahia. Bahia, 2016

\begin{tabular}{lrrr}
\hline \multicolumn{1}{c}{ Região da Bahia } & Número de municípios & $\begin{array}{c}\text { Número de municípios } \\
\text { com LRPD }\end{array}$ & Produtividade \\
\hline Centro-leste & $72(17,3 \%)$ & $5(9,8 \%)$ & $2.561(5,5 \%)$ \\
\hline Centro-norte & $38(9,1 \%)$ & $3(5,9 \%)$ & $1.067(2,3 \%)$ \\
Extremo Sul & $21(5,0 \%)$ & $3(5,9 \%)$ & $1.239(2,6 \%)$ \\
\hline Leste & $47(11,3 \%)$ & $7(13,7 \%)$ & $11.015(23,5 \%)$ \\
Nordeste & $33(7,9 \%)$ & $1(2,0 \%)$ & $3.562(7,6 \%)$ \\
\hline Norte & $28(6,7 \%)$ & $1(2,0 \%)$ & $8.168(17,4 \%)$ \\
\hline Oeste & $36(8,6 \%)$ & $3(5,9 \%)$ & $5.407(11,5 \%)$ \\
\hline Sudeste & $74(17,7 \%)$ & $5(9,8 \%)$ & $2.479(5,3 \%)$ \\
Sul & $68(16,3 \%)$ & $4(7,4 \%)$ & $11.372(24,3 \%)$ \\
\hline & $417(100 \%)$ & $51(100 \%)$ & $46.870(100 \%)$ \\
\hline
\end{tabular}

Fonte: SIA SUS. 
A tabela 2 apresenta o quantitativo de prótese produzido pelos LRPD no estado da Bahia, no ano de 2016 por tipo de prótese. A prótese total foi a mais realizada com $58,73 \%$, com destaque para a prótese total maxilar com uma produção de 15.635 unidades (33,36\%). A menor produtividade foi de prótese fixa unitária $(1,17 \%)$ e prótese sobre implante $(0,02 \%)$.

Tabela 2. Produtividade dos LRPD da Bahia por tipo de prótese dentária. Bahia, 2016

\begin{tabular}{lrr}
\hline \multicolumn{1}{c}{ TIPO DE PRÓTESE } & \multicolumn{2}{c}{ PRODUTIVIDADE } \\
\cline { 2 - 3 } & \multicolumn{1}{c}{$\mathrm{N}$} & $\%$ \\
\hline PT MAXILAR & 15635 & $33,36 \%$ \\
\hline PT MANDIBULAR & 11894 & $25,37 \%$ \\
\hline PPR MAXILAR & 8294 & $17,70 \%$ \\
\hline PPR MANDIBULAR & 10486 & $22,37 \%$ \\
\hline PF (UNITÁRIA) & 550 & $1,17 \%$ \\
\hline PRÓTESE SOBRE IMPLANTE & 11 & $0,02 \%$ \\
\hline TOTAL & 46870 & $100 \%$ \\
\hline FOnTP: SIA-SUS & &
\end{tabular}

Fonte: SIA-SUS.

Dos 51 municípios com LRPD, 33 (64,7\%) possuíam CEO e produziram 31.162 (67,3\%) próteses no ano de 2016, com média de 956,2 próteses por município. Os laboratórios localizados em municípios sem a presença de CEO (18) apresentaram uma média de produtividade de 850,8 próteses por município. Estes valores demonstram que não é grande a diferença na quantidade de próteses produzidas em municípios com e sem CEO (Tabela 3).

Tabela 3. Produtividade dos LRPD em municípios com e sem Centro de Especialidades Odontológicas. Bahia, 2016

\begin{tabular}{lrrr}
\hline & \multicolumn{2}{c}{ PRESENÇA DE CEO } & \multicolumn{1}{c}{ TOTAL } \\
\hline MUNICÍPIOS & \multicolumn{1}{c}{ SIM } & \multicolumn{1}{c}{ NÃO } & $51(100,0 \%)$ \\
PRODUTIVIDADE DE PRÓTESE & $33(64,7 \%)$ & $18(35,3 \%)$ & $46.870(100,0 \%)$ \\
MÉDIA DE PRODUTIVIDADE POR MUNICÍPIO & $31.556(67,3 \%)$ & $15.314(32,7 \%)$ & 919,0 \\
& 956,2 & 850,8 & $(+/-690,2)$ \\
\hline
\end{tabular}

Fonte: SIA-SUS.

\section{Discussão}

Os resultados deste estudo identificaram a produtividade de prótese dentária pelos LRPD da Bahia. Percebeu-se uma desigualdade na distribuição destes serviços por região, a quantidade de laboratórios que abrange cada região independe do número de municípios. Além disto, a região com maior produção de próteses dentárias foi a Sul (24,3\%), enquanto a menor produtividade foi na região Centro Norte (2,3\%), e estas regiões têm quantidade semelhante de LRPD, respectivamente 4 e 3 . Os laboratórios podem ter abrangência municipal ou regional e isto pode influenciar nos resultados de produtividade encontrados, pois laboratórios com abrangência regional fazem a cobertura de maior quantidade populacional e, consequentemente, podem ter uma maior produtividade.

Destaca-se que a produtividade dos serviços de saúde é influenciada não apenas pela quantidade dos serviços ofertados ou pela cobertura populacional, outros fatores como o planejamento e gestão do serviço, organização do processo de trabalho, referência e contrarreferência, acolhimento, vínculo e satisfação do usuário atuam como influentes no resultado da produtividade dos serviços e no cumprimento das metas pactuadas 10 月1

Guimarães et al. 月. contribui para o não credenciamento e para a perda do credenciamento dos laboratórios, principalmente em municípios com menor porte populacional. Sugere então como alternativa a implantação de laboratórios regionalizados, para garantir uma produção contínua das próteses, com distribuição dos custos fixos dos procedimentos entre os municípios envolvidos 
Ainda, Aguiar e Celeste Laboratórios Regionais de Prótese Dentária e de Técnicos em Prótese Dentária nas cinco regiões brasileiras, constatando desigualdades regionais na distribuição destes serviços. De acordo com estes autores, os aspectos geográficos, econômicos e culturais podem intervir na distribuição dos LRPD, tanto na sua oferta quanto no uso do serviçon.

Ressalta-se que no início da criação desta política, para o credenciamento destes estabelecimentos era utilizado um parâmetro populacional, em que, para cada grupo de 500.000 habitantes poderia ser cadastrado um LRPD por região de saúde. Em 2009, este parâmetro deixou de existir e os municípios com qualquer base populacional puderam requerer o credenciamento, sem restrições quanto à natureza jurídica dos estabelecimentos]. Essa desvinculação do credenciamento ao porte populacional dos municípios acarreta na maior possibilidade de oferta do serviço de prótese, evitando a distinção entre os municípios que podem ser beneficiados com o serviço e, consequentemente, universalizando o acesso.

O repasse financeiro aos municípios referente à produção de próteses dentárias ocorre de acordo com a faixa de produção/ mês pactuada. Dessa maneira, reitera-se a importância do acompanhamento dos LRPD, visto que a produção encontrada pode revelar problemas de gestão dos laboratórios, e não propriamente do município 1 .

Outro aspecto observado no presente estudo foi à carência de TPD e CD cadastrados nos LRPD, bem como uma distribuição desigual destes profissionais no território baiano. Verificou-se um total de 15 TPD, e apenas 3 CD cadastrados nos laboratórios identificados no CNES. Todavia, todos os LRPD deverão possuir, no mínimo, um Técnico de Prótese Dentária e/ ou um Cirurgião-Dentista, ambos com carga horária ambulatorial no SUS 5 . Uma hipótese para isto é que a mão de obra contratada pelos laboratórios pode não ser qualificada, assim os laboratórios não estão realizando o cadastro dos profissionais.
Guimarães et al.13 abordaram em seu estudo sobre os LRPD no estado de Minas Gerais que a baixa oferta de TPD foi o desafio mais frequente para a implantação dos laboratórios, contribuindo também para o descredenciamento dos LRPD. Evidenciando a necessidade de investimentos na formação desta categoria profissional. Além disso, apontaram a falta de capacidade técnica do CD como outro fator que contribuía para a manutenção dos LRPD, em alguns municípios, sendo necessário o envolvimento das instituições de ensino para garantir a capacitação dos recursos humanos do SUS envolvidos na confecção das próteses dentáriasilis.

Os LRPD podem oferecer somente o serviço de produção de próteses e serem classificados como isolados, ou podem estar inseridos em uma instituição de saúde, como Unidades Básicas de Saúde, Policlínicas, Hospitais, Centro de Especialidades Odontológicas, e serem classificados como serviço especializado da instituição em questão19. No que se refere a esta condição, neste estudo não houve diferença significativa no número de próteses realizadas em cidades com a existência ou não do CEO.

Quanto a descrição do tipo de prótese mais produzido nos LRPD do estado da Bahia, a prótese total teve a maior prevalência. Na comparação dos resultados do levantamento epidemiológico de saúde bucal no ano de 2003 e 2010 referente a avaliação da necessidade de próteses dentárias, constatou-se que nos adultos de 35 a 44 anos de idade houve aumento da necessidade de prótese no arco superior e redução no arco inferior e em idosos, houve um aumento na necessidade de prótese nos dois arcoseq. No Brasil, $15,4 \%$ dos idosos de 65 a 74 anos de idade necessitam de prótese total em ambos os arcos dentários e $17,9 \%$ necessitam de prótese total em um dos arcos 2 . Estas informações justificam os resultados encontrados no presente estudo referente ao tipo de próteses que são produzidas no estado da Bahia, com maior percentual para prótese total.

É pertinente ressaltar as limitações existentes nesta pesquisa, haja visto que a utilização de dados secundários não possibilita o domínio sobre equívocos que possam surgir por conta do preenchimento das informações e a não alimentação dos dados. 


\section{Considerações finais}

Embora haja uma quantidade considerável de próteses produzidas no estado da Bahia no ano de 2016, apenas $12,2 \%$ dos municípios baianos possuem laboratório de prótese dentária. A oferta de prótese dentária no SUS possibilita a universalização deste serviço, contribuindo com a melhoria da qualidade de vida relacionada a saúde bucal de toda a população. Assim, constata-se a necessidade de aumento do número de laboratórios de prótese e expansão da cobertura populacional deste serviço.

\section{Contribuições das autoras}

Alves FCS e Santos LPS realizaram a revisão de literatura, análise de dados e a redação do artigo científico. Oliveira NR e Guimarães LE realizaram a análise e discussão dos resultados. Oliveira NR realizou a revisão das referências e da redação final antes da submissão. Santos LPS realizou a revisão final.

\section{Conflitos de interesses}

Nenhum conflito financeiro, legal ou político envolvendo terceiros (governo, empresas e fundações privadas, etc.) foi declarado para nenhum aspecto do trabalho submetido (incluindo, mas não se limitando a subvenções e financiamentos, participação em conselho consultivo, desenho de estudo, preparação de manuscrito, análise estatística, etc.).

\section{Referências}

1. Brasil. Ministério da Saúde. Secretaria de Atenção Primária à Saúde. Departamento de Saúde da Familia. Coordenação-Geral de Saúde Bucal. Nota técnica: credenciamento e repasse de recursos para os Laboratórios Regionais de Próteses Dentárias LRPD. [Internet]. 2012. Disponível em: http://189.28.128.100/dab/ docs/geral/nota_tecnica_LRPD.pdf

2. Azevedo JS, Azevedo MS, Oliveira LJC, Correa MB, Demarco FF. Uso e necessidade de prótese dentária em idosos brasileiros segundo a Pesquisa Nacional de Saúde Bucal (SBBrasil 2010): prevalências e fatores associados. Cad Saúde Pública. 2017;33(8):e00054016. doi: 10.1590/0102-311X00054016

3. Barbato PR, Nagano HCM, Zanchet FN, Boing FN, Peres MA. Perdas dentárias e fatores sociais, demográficos e de serviços associados em adultos brasileiros: uma análise dos dados do estudo epidemiológico nacional. (Projeto SB Brasil 2002-2003). Cad Saude Publica. 2007;23(8):1803-14. doi: 10.1590/S0102311X2007000800007
4. Silva MES, Villaça EL, Magalhães CS, Ferreira EF. Impacto da perda dentária na qualidade de vida. Ciênc Saúde Coletiva. 2010;15(3):841-50. doi: 10.1590/S1413-81232010000300027

5. Brasil. Ministério da Saúde. Secretaria de Atenção à Saúde. Departamento de Atenção Básica. Coordenação Nacional de Saúde Bucal. Diretrizes da política nacional de saúde bucal. [Internet]. 2004. Disponíve em: http://189.28.128.100/dab/docs/ publicacoes/geral/diretrizes_da_politica_nacional_de_saude_bucal. pdf

6. Brasil. Ministério da Saúde. Portaria n. 1.570/GM, de 29 de julho de 2004. Estabelece critérios, normas e requisitos para a implantação e credenciamento de Centros de Especialidades Odontológicas e Laboratórios Regionais de Próteses Dentárias. Diário Oficial da União. 2004 jul. 29.

7. Brasil. Ministério da Saúde. Portaria n. 2.373, de 7 de outubro de 2009. Altera a redação da Portaria N 599/GM, de 23 de março de 2006. Diário Oficial da União. 2009 out. 7.

8. Brasil. Ministério da Saúde. Secretaria de Atenção Primária a Saúde. Cidades Atendidas com Laboratórios Regionais de Prótese Dentária. [Internet]. 2018. [citado em 2018 set. 12]. Disponível em: https://aps.saude.gov.br/ape/brasilsorridente/cidadesatendidas

9. Brasil. Ministério da Saúde. Brasil: Sistema de Informação Ambulatorial do SUS. [Internet]. 2018. Disponível em: http:// www2.datasus.gov.br/DATASUS/

10. Chaves SCL, Almeida AMFL, Rossi TRA, Santana SF, Barros SG, Santos CML. Política de Saúde Bucal no Brasil 2003-2014: cenário, propostas, ações e resultados. Ciênc Saúde Coletiva. 2017;22(6):1791-1803. doi: 10.1590/1413-81232017226.18782015

11. Aguiar VR, Celeste RK. Necessidade e alocação de laboratórios regionais de prótese dentária no Brasil: um estudo exploratório. Ciênc Saúde Coletiva. 2015;20(10):3121-8. doi: 10.1590/1413812320152010.18212014

12. Cunha MAGM, Matta-Machado ATG, Lucas SD, Abreu MHNG. Availability of Dental Prosthesis Procedures in Brazilian Primary Health Care. Biomed Research International. 2018;1-5. doi: $10.1155 / 2018 / 4536707$

13. Guimarães MRC, Pinto RS, Amaral JHL, Vargas AMD. Desafios para a oferta de prótese dentária na rede de saúde pública. Rev Odontol Unesp. 2017;46(1):39-44. doi: 10.1590/1807-2577.08716

14. Ribeiro CG, Cascaes AM, Silva AER, Seerig LM, Nascimento GG, Dermaco FF. Edentulism, severe tooth loss and lack of functional dentition in elders: a study in southern brazil. Braz Dent J. 2016;27(3):345-352. doi: 10.1590/0103-6440201600670

15. Brasil. Ministério da Saúde. Departamento de Atenção Básica. Coordenação Geral de Saúde Bucal. Nota técnica: credenciamento e repasse de recursos para os Laboratórios Regionais de Próteses Dentárias - LRPD. [Internet]. 2102. Disponível em: http://www. saude.pr.gov.br/arquivos/File/nota_tecnica_LRPD.pdf 
16. Santana DA, Santos LPS, Carvalho FS, Carvalho CAP.

Desempenho dos Centros de Especialidades Odontológicas de uma Região de Saúde da Bahia. Cad Saúde Colet. 2015;23(3):261267. doi: $10.1590 / 1414-462 \times 201400030122$

17. Viana IB, Moreira RS, Martelli PJL, Oliveira ALS, Monteiro IS. Avaliação da qualidade da assistência em saúde bucal na Atenção Primária à Saúde em Pernambuco, 2014. Epidemiol Serv Saúde. 2019;28(2):e2018060. doi: 10.5123/s1679-49742019000200015

18. Chaves SCL, Cruz DN, Barros SG, Figueiredo AL. Assessing the supply and use of secondary care in specialized dental clinics in Bahia State, Brazil. Cad Saúde Publica. 2011;27(1):143-154. doi: 10.1590/S0102-311X2011000100015

19. Brasil. Ministério da Saúde. Portaria $n^{\circ}$. 599, de 23 de março de 2006. Define a implantação de Especialidades Odontológicas (CEOs) 54 e de Laboratórios Regionais de Próteses Dentárias (LRPDs) e estabelecer critérios, normas e requisitos para seu credenciamento. Diário Oficial da União. 2006 mar. 23.

20. Colussi CF, Patel FS. Uso e necessidade de prótese dentária no Brasil: avanços, perspectivas e desafios. Sau \& Transf Soc. 2016;7(1):41-8.

21. Soares FF, Freire MCM, Reis SCGB. Projeto SB Brasil. Pesquisa Nacional de Saúde Bucal - Resultados Principais 2010. Comunic Saude Educ. 2018;22(64):981-9. doi: 10.1590/1807-57622015.0963 